\title{
Incidência de depressão e fatores associados em idosos residentes na comunidade: revisão de literatura
}

\author{
Incidence of depression and associated factors among elderly community-dwelling \\ people: a literature review

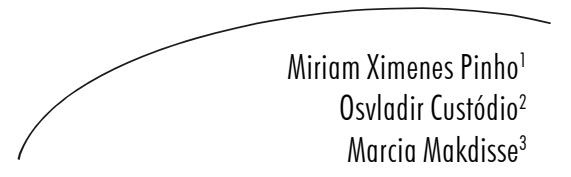

\section{Resumo}

Depressão geriátrica ocorre com frequência, mesmo na população residente na comunidade. Vários fatores de risco associados estão descritos na literatura. O objetivo deste estudo é realizar uma revisão da literatura sobre a incidência de depressão geriátrica e os fatores de risco associados em idosos residentes na comunidade. Para este fim, realizou-se revisão bibliográfica da literatura sobre o tema, sendo efetuada consulta às bases de dados MEDLINE, PUBMED, LILACS e SCIELO, utilizando-se das palavras chaves "idoso", "fator de risco", "depressão" e "incidência". A incidência de depressão na população idosa residente na comunidade é de 13,23\%, em média. Os preditores de depressão identificados foram: sexo feminino, idade avançada, condição marital, baixa escolaridade, condição socioeconômica desfavorável, condições de moradia, baixo suporte social, eventos estressores, depressão prévia, co-morbidades psiquiátricas, características de personalidade, distúrbios do sono, déficits cognitivos, condições de saúde adversas, limitação funcional e dor. Depressão ocorre com frequência na população idosa e constitui problema grave. A identificação dos fatores de risco associados com sua incidência pode ajudar os profissionais que atuam na área a diagnosticar e propor intervenções mais precoces e adequadas.

Universidade Federal de São Paulo, Escola Paulista de Medicina, Departamento de Medicina. São Paulo, SP, Brasil.

Universidade Federal de São Paulo, Escola Paulista de Medicina Departamento de Psiquiatria. São Paulo, SP, Brasil.

3 Hospital Israelita Albert Einstein, Divisão de Prática Médica/Programa de Cardiologia. São Paulo, SP, Brasil

Correspondência / Correspondence

Miriam Ximenes Pinho

Casa do Cardiopata da Escola Paulista de Medicina - Unifesp

Rua Otonis, 897 - V Clementino

04025-002 São Paulo, SP, Brasil

E-mail: miriampinho@yahoo.com
Palavras-chave:

Idoso. Fatores de risco. Depressão. Incidência.

Revisão 
Abstract

Depression in late life is a common psychiatric disorder among elderly community residents. Several risk factors have been described in literature. This study aimed to review the literature on the incidence of old age depression among community residents and the risk factors associated. A literature search was conducted in MEDLINE, LILACS and SCIELO databases using the following key words: "elderly", "risk factors", "depression", and "incidence". The incidence of late life depression in elderly people living in community varied from 5.4 to $24.1 \%$ (median: 12,0; interquartil range: 12,5). The main predictors of depressive disorders and depressive symptoms cases were: female sex, advanced age, marital condition, low educational status, socio-economic deprivation, living condition, low social support, life events, previous depression, psychiatry comorbidity, personality characteristics, sleep disorders, cognitive impairments, poor health conditions, functional limitations and pain. Depression is a common condition among community-living elderly people and it is considered a serious issue. The identification of factors associated with the incidence of depression may help health professionals to offer early diagnosis and more accurate therapeutic intervention.
Key words: Aged. Risk factors. Depression. Incidence.

Review. Elderly
INTRODUÇÃO

"A morte não é a maior tragédia do ser humano, é pior quando algo vital dentro da pessoa morre enquanto ela ainda está viva. Essa morte é certamente a coisa mais temível e trágica" (Daisaku Ikeda)*

Em 2000, os idosos brasileiros constituíam 14,5 milhões de pessoas, $8,6 \%$ da população total do país. ${ }^{1} \mathrm{O}$ último relatório de Síntese dos Indicadores Sociais, ${ }^{2}$ com base nos dados da PNAD de 2004, salienta que, atualmente, o Brasil ocupa a oitava posição mundial em idosos com mais de 60 anos, contando com 17,6 (9,7\%) milhões de pessoas e projeta que, em 2050 , terá 34,3 milhões de pessoas. Os maiores contingentes nesta faixa são encontrados nos estados de São Paulo, com 4 milhões, e Rio de Janeiro, com 2 milhões.

O índice de envelhecimento da população do país - uma razão entre as pessoas com 65 anos ou mais e aqueles com menos de 15 anos - era de 0,11 na década de 80 e saltou para 0,25 em pouco mais de 20 anos. Apesar de ainda ser considerado um país jovem, a população brasileira está envelhecendo, ${ }^{2}$ em paralelo ao fenômeno mundial.

\footnotetext{
* Daisaku Ikeda, filósofo, escritor, educador e líder budista japonês.
} 
A proporção de indivíduos com 80 ou mais anos está crescendo e, hoje, já representa $12,6 \%$ da população idosa brasileira, o que torna heterogênea a composição etária dentro do grupo de idosos. ${ }^{3}$

Por conta da esperança de vida menor, entre os idosos, o número de homens é menor que o de mulheres é estimado que, atualmente, para 81,5 homens há $100 \mathrm{mu}-$ lheres vivas nesta faixa etária. Esta diferença tende a se acentuar com o avançar da idade. $\mathrm{Na}$ faixa etária com 80 ou mais anos, por exemplo, as mulheres representam $60 \%$ da população idosa. ${ }^{3}$ Em consequência, de acordo com Papaléo Netto, ${ }^{4}$ as mulheres "estão expostas porperiodos mais longos às doenças crônico-degenerativas, à viuvez e à solidão".

O rápido crescimento da população idosa no país tem fomentado investimentos na área de políticas públicas de saúde e pesquisas, além de gerar demanda crescente por capacitação especializada por parte dos profissionais interessados em atuar ou que já atuam junto a essa população.

A expectativa é de que os cuidados em saúde geriátrica e gerontológica estejam entrando em uma nova era de crescimento e desenvolvimento, apesar de inúmeros problemas de infra-estrutura em diversos setores ainda persistirem em nosso país. Permanecem, portanto, os desafios. ${ }^{5}$

A população idosa apresenta maior vulnerabilidade para problemas de saúde que a população geral, e utiliza com maior frequência os serviços de saúde. A presença de transtornos mentais nessa faixa etária também é alta; em um estudo, a internação psiquiátrica aparecia entre as dez principais causas de internação entre idosos do sexo masculino..$^{6-8}$

Os principais transtornos mentais presentes na população idosa são os demenciais e os depressivos. ${ }^{7,8}$ A depressão ocupa o quinto lugar como o problema de saúde mais prevalente no mundo, afetando 120 milhões de pessoas, segundo a OMS. De acordo com as projeções, estima-se que, em 20 anos, a depressão ocupará o $2^{\circ}$ lugar, perdendo apenas para as doenças cardíacas.

\section{Depressão: caracterização e diagnóstico}

Não é fácil conceituar ou mesmo diagnosticar depressão, devido à multiplicidade de suas manifestações e da extensão de sua nosografia. ${ }^{7,10,11}$

De acordo com Del Porto, ${ }^{12}$ o termo depressão gera enorme confusão diagnóstica entre os profissionais de saúde, por conta dos inúmeros significados que o termo tem - por exemplo, um estado afetivo normal (tristeza), um sintoma, uma síndrome e uma doença. Esse autor propôs algumas definições para reduzir a confusão e melhorar a comunicação entre profissionais:

Sentimento de tristeza é uma resposta normal e adaptativa do ser humano diante de situações adversas (perda, derrota, desapontamentos) e pode ser um momento de reflexão e de preparação para novas ações 
no futuro. A tristeza não deve se constituir em alvo de intervenção médica e nem em diagnóstico psiquiátrico.

Sintoma depressivo pode surgir nos mais variados quadros clínicos (transtorno de estresse pós-traumático, demências, esquizofrenia, alcoolismo, doenças clínicas, etc). Também pode ocorrer como resposta a estressores psicossociais.

Síndrome depressiva inclui alterações de humor (tristeza, irritabilidade, falta de capacidade de sentir prazer, apatia) e de outros aspectos, tais como alterações cognitivas, psicomotoras e vegetativas.

Depressão como doença é classificada de várias formas, de acordo com o período histórico, a preferência dos autores e o referencial teórico adotado. Entre os principais quadros mencionados atualmente, temos: transtorno depressivo maior, menor, distimia, depressão integrante do transtorno bipolar, etc.

A característica mais típica dos quadros depressivos é a presença de queixas referentes à sensação de tristeza e vazio. Porém, nem todos os pacientes apresentam queixas de humor triste ou deprimido, ao invés disso podem referir fadiga, dificuldades de concentração e lentificação psicomotora. ${ }^{12}$

O diagnóstico dos transtornos depressivos pode ser operacionalizado por meio dos critérios diagnósticos disponíveis nas classificações internacionalmente reconhecidas: o Manual Diagnóstico e Estatístico de
Transtornos Mentais (DSM-IV-TR) ${ }^{13}$ e a Classificação Internacional de Doenças (CID-10), ${ }^{14}$ da OMS. A vantagem do uso desses manuais classificatórios, tanto na prática clínica quanto em pesquisas, é o uso de uma linguagem em comum, favorecendo a comunicação entre profissionais da área de saúde mental.

$\mathrm{Na}$ literatura, o transtorno depressivo mais bem estudado é a depressão maior, a forma mais grave. De acordo com o DSMIV (1994), ${ }^{15}$ a característica essencial de um episódio depressivo maior é o humor deprimido ou perda de interesse ou prazer por quase todas as atividades, por um período mínimo de duas semanas. O indivíduo também deve experimentar pelo menos quatros dos sintomas a seguir: (1) perda ou ganho de peso significativo; (2) insônia ou hipersonia; (3) agitação ou retardo psicomotor; (4) fadiga ou perda de energia; (5) culpa e autodesvalorização; (6) diminuição da capacidade de pensar e concentrar; (7) pensamentos recorrentes de morte, tentativa ou ideação suicida.

Já o transtorno depressivo menor é descrito de forma idêntica à depressão maior, diferenciando-se apenas por envolver menos sintomas (dois a quatro sintomas) e menor prejuízo. ${ }^{15}$

Outra forma de depressão frequentemente citada é a distimia, cuja característica essencial é a presença de humor deprimido crônico, presente por pelo menos dois anos. Os sintomas depressivos no transtorno distímico não costumam ser tão graves e inca- 
pacitantes quanto aqueles presentes na depressão maior. ${ }^{15}$

\section{Depressão no idoso}

A depressão tem sido apontada como um problema de saúde pública que afeta pelo menos um em cada seis pacientes idosos tratados na atenção básica. ${ }^{16}$

As taxas de prevalência de depressão geriátrica diferem consideravelmente, dependendo da definição de depressão, do critério diagnóstico e da população de interesse, sendo mais altas onde comorbidades com doenças físicas são mais comuns. ${ }^{17,18}$ As estimativas de prevalência de todas as formas de depressão geriátrica são mais altas nos pacientes institucionalizados intactos cognitivamente (cerca de 60\%), intermediárias nos pacientes com doenças crônicas hospitalizados ou em unidades de atenção primária (cerca de $25 \%$ ) e mais baixas nos residentes na comunidade (cerca de 10\%). ${ }^{16}$

Depressão geriátrica não é frequentemente diagnosticada e, mais importante, não é tratada. Os inúmeros problemas sociais e econômicos dos idosos, adicionados à progressiva debilidade física, levam muitos profissionais de saúde a concluir que depressão é uma consequência normal desses problemas - atitude muitas vezes compartilhada pelos próprios idosos que, em geral, raramente se queixam ou utilizam o termo depressão, mas apresentam queixas somáticas vagas e inespecíficas que podem estar mascarando um transtorno depressivo. . $^{19,20}$
Quando, ainda, a depressão é secundária a doenças físicas que apresentam mal-estar, apatia, fadiga, distúrbios de memória ou concentração como manifestações, seu diagnóstico é mais difícil. ${ }^{71,21} \mathrm{~A}$ influência do próprio processo de envelhecimento no surgimento, curso e desfecho das depressões ainda não está clara. Por outro lado, há certo consenso de que os aspectos biológicos, sociais e psicológicos do indivíduo exercem papel importante no desencadeamento e manutenção dos quadros depressivos. ${ }^{7}$

A depressão tem consequências graves, incluindo sofrimento dos pacientes e cuidadores, piora da incapacidade associada à doença física e aos transtornos cognitivos, aumento dos custos dos cuidados de saúde e mortalidade aumentada relacionada ao suicídio e à doença física. ${ }^{16}$

Depressão geriátrica está associada a inúmeros fatores de risco descritos tanto em estudos de corte transversal quanto em estudos prospectivos. Os fatores mais identificados são: sexo feminino, idade, viuvez, baixa escolaridade e renda, presença de eventos de vida estressores, baixo suporte social, características de personalidade, percepção de baixa qualidade de vida e condições de saúde, presença de déficits cognitivos, limitação funcional, histórico psiquiátrico e comorbidades psiquiátricas, uso e abuso de álcool, uso de fármacos (digoxina, inibidores da enzima de conversão da angiotensina, bloqueadores do canal de cálcio, betabloqueadores), presença de doenças físicas agudas e crônicas, dor e comorbidades..$^{22-26}$ 
O objetivo deste estudo foi realizar uma revisão da literatura sobre a incidência de depressão e os fatores de risco associados em idosos residentes na comunidade.

\section{METODOLOGIA}

Trata-se de estudo exploratório realizado por meio de um levantamento bibliográfico junto às bases de dados Medline, LILACS e Scielo.

Utilizaram-se, para a busca, as seguintes palavras-chaves: aged/idoso/ anciano, risk. factors/ fatores de risco/factores de riesgo, depression/ depressão/depressión. Nesta busca, cerca de 140 estudos publicados foram identificados nos últimos dez anos (1997-2007) em inglês, português e espanhol. Nesta fase, foi realizada leitura exploratória, com o reconhecimento do material que atenderia aos critérios de inclusão do estudo.

Conforme salientado, as taxas de prevalência de depressão diferem entre os estudos, em função da definição de depressão, do critério diagnóstico e da população de interesse - por isso, alguns critérios de inclusão e exclusão foram adotados para uniformização dos resultados. Só foram incluídos estudos longitudinais prospectivos, utilizando critérios válidos e confiáveis para o diagnóstico de depressão, com pessoas residentes na comunidade e com mais 50 anos. Os que apresentavam outros transtornos psiquiátricos sobrepostos (ansiedade, transtorno bipolar, psicose, etc.) foram excluídos. Nesta fase, realizou-se leitura seletiva para a escolha do material, que serviria aos propósitos do estudo; observaram-se o tipo de estudo, o método de seleção da amostra, tamanho da amostra e os instrumentos utilizados. Após a leitura dos 140 estudos, restaram apenas 11 em língua inglesa.

A partir dos 11 estudos, foram feitas as leituras finais. Numa leitura - a analítica os resultados foram extraídos e lançados em tabelas, com o intuito de favorecer a compreensão e a ilustração. Finalmente, uma leitura analítica interpretativa foi realizada para a avaliação crítica dos resultados e a construção da discussão.

\section{RESULTADOS}

A descrição das características dos estudos encontra-se ilustrada, por ordem cronológica, na tabela 1. A incidência de depressão e os fatores de risco associados nos estudos prospectivos estão descritos nas tabelas 2, 3 e 4 . Somente os fatores que atingiram significância estatística $(\leq 0,05)$ foram incluídos. 
Tabela 1 - Características gerais dos 11 estudos analisados. São Paulo, SP, 2007.

\begin{tabular}{|c|c|c|c|c|c|c|c|}
\hline \multirow[t]{2}{*}{$\mathrm{N}^{\mathrm{o}}$. } & \multirow[t]{2}{*}{ Estudo } & \multirow[t]{2}{*}{ Local } & \multicolumn{2}{|r|}{$\mathrm{n}$} & \multirow{2}{*}{$\begin{array}{l}\text { Faixa de } \\
\text { Idade } \\
\text { (média) }\end{array}$} & \multirow{2}{*}{$\begin{array}{c}\text { Mulheres } \\
\%\end{array}$} & \multirow{2}{*}{$\begin{array}{c}\text { Extensão } \\
\text { do estudo } \\
\text { (meses) }\end{array}$} \\
\hline & & & Início & Follow -up & & & \\
\hline 1 & Roberts et al, $1997^{27}$ & Califórnia, EUA & 2.219 & 2.219 & $\begin{array}{l}50-95 \\
(64,7)\end{array}$ & 66,3 & 12 \\
\hline 2 & Prince et al, $1998^{28}$ & Londres, Inglaterra & 654 & 451 & $\begin{array}{r}65-98 \\
(75,8)\end{array}$ & 61 & 12 \\
\hline 3 & Turvey L L, $1999^{29}$ & Michigan, EUA & - & 5.449 & $70-103(77)$ & 62 & 24 \\
\hline 4 & $\begin{array}{l}\text { Geerlings L L, } \\
2000^{30}\end{array}$ & $\begin{array}{l}\text { Amsterdam, } \\
\text { Holanda }\end{array}$ & 652 & 525 & $55-85(69)$ & 57,7 & 36 \\
\hline 5 & $\begin{array}{l}\text { Schoevers et al, } \\
2000^{31}\end{array}$ & Amsterdam, Holanda & 3.747 & 1.940 & $\begin{array}{l}65-84 \\
(74,5)\end{array}$ & 62,4 & 36 \\
\hline 6 & $\begin{array}{l}\text { Livingston L L, } \\
2000^{32}\end{array}$ & Londres, Inglat erra & 141 & 79 & $65-95(78)$ & 77,2 & 36 \\
\hline 7 & Roberts et al, $2000^{33}$ & Califórnia, EUA & 2370 & 2228 & $\begin{array}{r}50-95 \\
(65)\end{array}$ & 56,37 & 12 \\
\hline 8 & Beekman et al, $2001^{34}$ & $\begin{array}{l}\text { Amsterdam, } \\
\text { Holanda }\end{array}$ & 3056 & 2200 & $55-85(69)$ & 53 & 36 \\
\hline 9 & $\begin{array}{l}\text { Geerlings L L, } \\
2001^{35}\end{array}$ & $\begin{array}{l}\text { Amsterdam, } \\
\text { Holanda }\end{array}$ & 652 & 525 & $55-85(69)$ & 57,7 & 36 \\
\hline 10 & Heun, Heun, $2005^{36}$ & $\begin{array}{l}\text { Bonn e } \\
\text { Mainz,Alemanha }\end{array}$ & 1582 & 1431 & $55-?(61)$ & 55 & 52 \\
\hline 11 & Harris L L, $2006^{37}$ & Londres, Inglaterra & 1552 & 1164 & $\begin{array}{r}65-104 \\
(84,5) \\
\end{array}$ & 61,25 & 24 \\
\hline
\end{tabular}

\section{Incidência de depressão}

A mediana de incidência total de casos de depressão e sintomas depressivos foi $12 \%$ (amplitude interquartil 12,5), e os extremos da distribuição foram 5,4- 24.1\% (tabela 2). Esses estudos foram conduzidos em indivíduos com idade entre 50 e 104 anos, residentes na comunidade, nos Estados Unidos, Inglaterra, Holanda e Alemanha (tabela 1)..$^{27-37}$

Apenas dois estudos ${ }^{27,36}$ avaliaram a incidência de depressão maior, como definido pelos critérios diagnósticos do DSM, e apresentaram resultados bastante diferentes. Um estudo americano ${ }^{27}$ estimou a incidência de $5,4 \%$, enquanto um conduzi- do, ${ }^{36}$ na Alemanha, estimou uma taxa quatro vezes maior.

A presença de sintomas depressivos foi investigada em sete estudos e a mediana da incidência foi 12,0 (amplitude interquartil 7,5), com extremos de distribuição de 6,0$24,1 \%$ (tabela 2). Foram utilizados diferentes instrumentos nesses estudos: GDS-15, PRIME-MD, Short-CARE, CES-D, GMSAGECAT, CIDI. ${ }^{27-37}$

A mediana dos três estudos conduzidos na Inglaterra foi $12,028,32,37 \mathrm{e}$ a dos estudos realizados na Holanda, 14,3.30,31,34 Porém, foi observada maior variabilidade entre resultados nos estudos ingleses do que nos estudos holandesas. 
Tabela 2 - Incidência de depressão e características dos estudos quanto aos instrumentos e critérios usados para avaliar depressão ou sintomas depressivos. São Paulo, SP, 2007.

\begin{tabular}{|c|c|c|c|c|}
\hline No. & Estudo & Instrumento & Critério de depressão & $\begin{array}{c}\text { Incidência de } \\
\text { depressão }\end{array}$ \\
\hline 1 & Roberts et al, $1997{ }^{27}$ & PRIME-MD ${ }^{a}$ & Episódio depressivo maior/DSM-IV & 5,4 \\
\hline 2 & Prince et al, $1998{ }^{28}$ & Short-CARE ${ }^{b}$ & Depressão pervasiva $^{c}$ & 12 \\
\hline 3 & Turvey et al, 199929 & CES-D ${ }^{d}$ modificada & Síndrome depressiva & 6 \\
\hline 4 & Geerlings et al, $2000^{30}$ & CES-D & Síndrome depressiva & 14,1 \\
\hline 5 & Schoevers et al, $2000{ }^{31}$ & GMS-AGECAT $^{\mathrm{e}}$ & Síndrome depressiva & 15,9 \\
\hline 6 & Livingston et al, $2000^{32}$ & Short-CARE/ DPHS ${ }^{f}$ & Síndrome depressiva & 24,1 \\
\hline 7 & Roberts et al, $2000^{33}$ & PRIME-MD & Episódio depressivo maior (DSM-IV) & - \\
\hline 8 & Beekman et al, $2001^{34}$ & CES-D & Síndrome depressiva & 9,7 \\
\hline 9 & Geerlings et al, $2001{ }^{35}$ & CES-D & Síndrome depressiva & - \\
\hline 10 & Heun, Heun, $2005^{36}$ & $\mathrm{CIDI}^{\mathrm{g}}$ & Episódio depressivo maior/DSM-III-R & 23,5 \\
\hline 11 & Harris et al, $2006^{37}$ & GDS- $15^{\mathrm{h}}$ & Síndrome depressiva & 8,4 \\
\hline
\end{tabular}

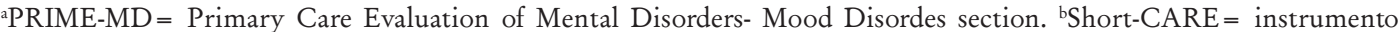
usado para avaliar depressão, demência e incapacidades. cestado depressivo de moderado a grave capaz de afetar múltiplas funções e áreas da vida do indivíduo. ${ }^{\mathrm{d}} \mathrm{CES}-\mathrm{D}=$ Center for Epidemiologic Studies Depression Scale. ${ }^{\mathrm{e}} \mathrm{GMS}-$ AGECAT $=$ Geriatric Mental State Examination-itens. Avalia síndromes orgânicas, afetivas e ansiosas. ${ }^{\mathrm{f} D P H S}=$ Depression Homogenous Scale. ${ }^{\mathrm{g}} \mathrm{CIDI}=$ Composite International Diagnostic Interviiew. ${ }^{\mathrm{h}} \mathrm{GDS}-15=$ Geriatric Depression Scale-15 itens.

Fatores de risco associados com depressão geriátrica

Os fatores de risco encontrados foram agrupados em fatores sócio-demográficos, suporte social, eventos de vida estressores, morbidades psiquiátricas e condições de saúde:

\section{(a) Fatores sócio-demográficos}

- Gênero. Em alguns estudos, a presença de depressão e sintomas depressivos estava associada com mais frequência ao sexo feminino. ${ }^{27,30,33,34,36}$ Em outros, não foi observada a associação de depressão com sexo. ${ }^{28,29,31,32} \mathrm{E}$, num único estudo, sexo feminino foi fator protetor para depressão. ${ }^{37}$
- Idade. Na maioria dos estudos, houve aumento da presença de depressão e sintomas depressivos em indivíduos nas faixas mais elevadas de idade, independentemente do sexo. ${ }^{27,30,31,33,3,3,36,37}$ Dois estudos não constataram associação entre a idade e o risco de depressão. ${ }^{28,29} \mathrm{Um}$ único estudo demonstrou que indivíduos mais jovens ( $\leq 78$ anos) apresentavam risco maior de desenvolver depressão. ${ }^{32}$

- Situacão conjugal. Os idosos casados apresentaram risco menor de desenvolver quadros depressivos do que aqueles que não eram mais ou nunca foram casados. ${ }^{28,30,34}$ Prince et al. ${ }^{28}$ estratificaram por sexo e observaram que as mulheres casadas tinham maior risco que os homens de ficarem deprimidas, porém, entre os solteiros, os ho- 
mens apresentaram maior risco que as mulheres. Três outros estudos não evidenciaram a associação do status marital e o aparecimento de depressão ou sintomas depressivos. ${ }^{27,31,33}$

- Escolaridade. Baixo nível de escolaridade foi associado com depressão em três estudos, indicando que quanto maior o nível educacional, menor o risco de apresentar quadros depressivos. ${ }^{30,33,34}$ Em quatro estudos, a associação de escolaridade com depressão não foi encontrada. ${ }^{27,31,32,36}$

- Condição socioeconômica. A presença de problemas financeiros foi associada com depressão em três estudos. ${ }^{27,33,36}$ Outros dois não encontraram associação de renda e status socioeconômico com depressão. ${ }^{28,31}$

- Condições de moradia. Presença de condições adversas de moradia (violência, trânsito, barulho, transporte público insuficiente ou inadequado, falta de saneamento), ${ }^{27}$ assim como morar em cidade grande ${ }^{34}$ apareceram associados com depressão.

\section{(b) Suporte social}

Pouco ou nenhum contato com amigos, vizinhos ou familiares foi associado com maior risco de depressão. ${ }^{27,28,34}$ Insatisfação com o suporte recebido, passar muito tempo só, sentir-se só e desavenças nos relacionamentos pessoais também foram associados com a presença de depressão. ${ }^{28,33,34,37} \mathrm{Em}$ dois estudos, o suporte social não apresentou nenhum efeito no aparecimento de depressão. ${ }^{30,31}$ (c) Eventos de vida estressores

A ocorrência de eventos estressores psicossociais, nos últimos três anos foi associada com o surgimento de depressão. ${ }^{29,31,32,37} \mathrm{Os}$ principais estressores psicossociais foram a perda do parceiro (viuvez), surgimento de doenças e incapacidades, doença de familiar e institucionalização. ${ }^{27,29,31,32}$ Um estudo destacou que a perda do parceiro associou-se com depressão, especialmente entre os viúvos recentes. ${ }^{29}$ Presença de três ou mais estressores $(\geq 3)$ na vida do indivíduo também se associou ao aparecimento de depressão. ${ }^{27}$

Prince et $a l .^{28}$ não observaram aumento de risco para depressão na presença de estressores psicossociais (viuvez ou ter doença grave) ou entre aqueles que relataram ocorrência de mais de dois eventos.

(d) Morbidades psiquiátricas

- Histórico psiquiátrico prévio e familiar. Episódio depressivo prévio foi importante preditor de depressão em quatro estudos. ${ }^{27,31,36,37}$ Turvey et al. ${ }^{29}$ não observaram diferenças entre deprimidos e não-deprimidos quanto à presença de histórico psiquiátrico prévio nos pacientes que perderam o cônjuge.

Apenas um estudo avaliou a vulnerabilidade individual para depressão com base na história psiquiátrica familiar e não observou nenhuma associação. ${ }^{31}$

- Comorbidadespsiquiátricas. A presença de ansiedade foi observada em deprimidos em três estudos. ${ }^{31,36,37}$ Heun e Hein ${ }^{36}$ também 
observaram coexistência de depressão com transtornos somatoformes e demenciais.

- Personalidade. Livingston et al..$^{32}$ observaram presença de traços de neuroticismo mais frequentemente em indivíduos deprimidos. Beekman et al. ${ }^{34}$ encontraram que os deprimidos apresentavam maior prevalência do traço "locus de controle externo", ou seja, sentiam-se mais vítimas do destino do que no controle de suas próprias vidas. Já Harris et al. ${ }^{37}$ não encontraram associação do traço "locus de controle" com depressão.

- Abuso ou dependência do uso de álcool. Os estudos que consideraram o consumo de álcool como fator de risco para depressão não evidenciaram a associação. $32,33,36$

- Distúrbios do sono. Em dois estudos, queixas em relação à quantidade de sono (insônia, hipersonia, outras queixas) associaramse com o risco aumentado de depressão. ${ }^{28,33}$

- Déficits cognitivos. Dois estudos observaram que déficits cognitivos leves ou moderados avaliados a partir do mini-exame do estado mental foram associados com o aparecimento de depressão. ${ }^{34,36}$ Alguns estudos não constataram tal associação..$^{28,31}$

Queixas subjetivas de perda de memória associaram-se com o surgimento de depressão..$^{27,36}$

(e) Condições de saúde

Presença de doenças crônicas (por exemplo, hipertensão arterial, doença cardíaca, doença pulmonares, artrite, diabetes) asso- ciou-se de forma significativa com o aparecimento de depressão em cinco estudos. $^{27,28,30,31,34}$

Livingston $e t$ al..$^{32}$ verificaram a utilização de serviços médicos e não observaram aumento de consultas por pacientes deprimidos com condições médicas crônicas em relação aos não-deprimidos. No mesmo estudo, os pacientes com doenças agudas ficaram deprimidos com mais frequência do que aqueles com doenças crônicas.

A presença concomitante de mais de uma doença crônica foi associada com a incidência de depressão em cinco estudos. ${ }^{27,28,30,31,37}$

Em dois estudos, o aparecimento de depressão foi observado nos pacientes que haviam apresentado condição de saúde mais grave ou naqueles que se consideravam com pior condição de saúde. ${ }^{28,37}$

Limitação funcional. Limitações na realização de atividades de vida diária (pessoais e instrumentais) associaram-se com o surgimento de depressão na maioria dos es$\operatorname{tudos}^{27-31,34,37}$ e, quanto mais graves estas limitações, maiores foram as incidências observadas. $^{27,28,30,34,37}$

Prince et al. ${ }^{28}$ avaliaram especificamente os prejuízos resultantes de doenças ou incapacidades, que limita ou impede o desempenho de papéis do indivíduo, e observaram que estes prejuízos se associavam à incidência aumentada de depressão. Um estudo não encontrou associação entre limitação funcional e depressão. ${ }^{32}$ 
Dor. Em quatro estudos, o surgimento de depressão apareceu associado com a frequência e a intensidade da dor. ${ }^{28,32,35,37}$
Geerlings et al. ${ }^{35}$ observaram que nos casos mais graves de sintomas depressivos (escala CES-D), a associação com dor era maior nos homens do que nas mulheres.

Tabela 3 - Fatores de risco associados com incidência de depressão nos estudos prospectivos. São Paulo, SP, 2007.

\begin{tabular}{|c|c|c|c|c|}
\hline Estudo & Fatores de Risco & OR & IC & $\mathrm{p}$ \\
\hline \multirow{10}{*}{$\begin{array}{l}\text { 1. Roberts et al, } \\
1997^{27}\end{array}$} & - Idade avançada ( $\geq 80$ anos) & 2.35 & $1.28-2.60$ & 0.006 \\
\hline & - Sexo feminino & 1.71 & $1.14-2.57$ & 0.01 \\
\hline & - Problemas financeiros & 1.83 & $1.15-2.92$ & 0.01 \\
\hline & - Doenças crônicas $(\geq 2)$ & 2.97 & $1.86-4.75$ & $<0.0001$ \\
\hline & - Limitação funcional & 3.35 & $2.02-5.57$ & $<0.0001$ \\
\hline & - Déficits cognitivos & 3.87 & $2.19-6.83$ & $<0.0001$ \\
\hline & - Eventos de vida $(\geq 3)$ & 1.78 & $1.06-2.97$ & 0.03 \\
\hline & - Problemas na qualidade da vizinhança & 2.85 & $1.78-4.56$ & $<0.0001$ \\
\hline & - Isolamento social & 2.51 & $1.55-4.06$ & 0.0002 \\
\hline & - Episódio depressivo prévio & 14.91 & $10.59-20.99$ & $<0.0001$ \\
\hline \multirow{12}{*}{$\begin{array}{l}\text { 2. Prince et al, } \\
1998^{28}\end{array}$} & - Deficiência ${ }^{a}$ (Pressão alta) & 5.1 & $1.6-16.3$ & $\mathrm{ND}^{*}$ \\
\hline & - Deficiência (falta de ar) & 3.4 & $1.8-6.5$ & ND \\
\hline & - No. deficiências $(\geq 3)$ & $3.5(\mathrm{RR})$ & $1.7-7.3$ & ND \\
\hline & - Distúrbio do sono & 3.1 & $1.6-5.9$ & ND \\
\hline & - Dor & 3.9 & $1.9-8.1$ & ND \\
\hline & - Suporte social (falta de contato c/ amigos) & $2.5(\mathrm{RR})$ & $1.3-3.7$ & ND \\
\hline & - Suporte social (sentir-se só) & $3.6(\mathrm{RR})$ & $2.0-6.4$ & ND \\
\hline & - Ser separado/divorciado & $3.5(\mathrm{RR})$ & $1.6-7.7$ & ND \\
\hline & - Ser casado (mulheres) & 6.3 & $1.3-29.7$ & ND \\
\hline & - Gravidade da doença & $2.7(\mathrm{RR})$ & 1.3-5.6 & ND \\
\hline & - Dependência funcional (AVD’s) ${ }^{b}$ & 3.3 & $1.6-6.6$ & ND \\
\hline & - Desvantagem ${ }^{c}$ resultante de doenças & 3.9 & $1.36-11.4$ & ND \\
\hline $\begin{array}{l}\text { 3. Turvey et al, } \\
1999^{29}\end{array}$ & - Eventos de vida (viuvez) & 3.8 & 2.1-6.7 & $<0.0001$ \\
\hline \multirow{7}{*}{$\begin{array}{l}\text { 4. Geerlings et al, } \\
2000^{30}\end{array}$} & - Sexo feminino & 3.22 & $1.43-7.28$ & ND \\
\hline & - Idade avançada & 1.07 & $1.02-1.12$ & ND \\
\hline & - Escolaridade Baixa & 3.08 & $1.44-6.56$ & ND \\
\hline & - Não ser casado & 2.38 & $1.12-5.03$ & ND \\
\hline & - Doenças crônicas (>1) & 4.06 & $1.41-11.67$ & ND \\
\hline & - Limitação funcional & 5.90 & $2.13-16-34$ & ND \\
\hline & - Limitação das atividades resultante de doença & 2.37 & $1.05-5.33$ & ND \\
\hline \multirow{9}{*}{$\begin{array}{l}\text { 5. Schoevers et al, } \\
2000^{31}\end{array}$} & - Idade avançada ( $\geq 74$ anos) & 1.30 & $1.06-1.60$ & ND \\
\hline & - Episódio depressivo prévio & 1.61 & $1.25-2.06$ & ND \\
\hline & - Doenças crônicas & 1.46 & $1.19-1.90$ & ND \\
\hline & - Novas doenças crônicas & 1.40 & $1.11-1.77$ & ND \\
\hline & - Limitação funcional & 1.55 & $1.26-1.92$ & ND \\
\hline & - Piora da limitação funcional & 1.73 & $1.39-2.14$ & ND \\
\hline & - Eventos de vida (morte do companheiro/a) & 2.30 & $1.77-2.99$ & ND \\
\hline & - Comorbidade psiquiátrica (ansiedade) & 2.56 & $1.57-4.19$ & ND \\
\hline & - Comorbidade orgânica & 1.57 & $1.13-2.17$ & ND \\
\hline \multirow{4}{*}{$\begin{array}{l}\text { 6. Livingston et al, } \\
2000^{32}\end{array}$} & - Dor frequente & ND & ND & 0.02 \\
\hline & - Doença aguda recente & ND & ND & 0.05 \\
\hline & - Eventos de vida (doença em familiar) & ND & ND & 0.02 \\
\hline & - Personalidade (neuroticismo) ${ }^{\mathrm{d}}$ & ND & ND & 0.01 \\
\hline
\end{tabular}


Tabela 3 - Fatores de risco associados com incidência de depressão nos estudos prospectivos. São Paulo, SP, 2007. (continuação)

\begin{tabular}{|c|c|c|c|c|}
\hline Estudo & Fatores de Risco & OR & IC & $\mathrm{p}$ \\
\hline \multirow{7}{*}{$\begin{array}{l}\text { 7. Roberts et al. } \\
, 2000^{33}\end{array}$} & - Distúrbio do sono & 3.32 & $2.29-4.83$ & ND \\
\hline & - Idade avançada ( $\geq 80$ anos) & 1.93 & $1.06-3.50$ & ND \\
\hline & - Sexo feminino & 1.69 & $1.14-2.50$ & ND \\
\hline & - Baixo suporte social & 2.78 & $1.74-4.43$ & ND \\
\hline & - Escolaridade baixa & 1.62 & $1.03-2.57$ & ND \\
\hline & - Problemas financeiros & 2.00 & $1.30-3.08$ & ND \\
\hline & - Limitação funcional & 3.09 & $1.88-5.06$ & ND \\
\hline \multirow{10}{*}{$\begin{array}{l}\text { 8. Beekman et al, } \\
2001^{34}\end{array}$} & - Sexo feminino & ND & ND & ND \\
\hline & - Idade avançada & ND & ND & ND \\
\hline & - Não ser casado & ND & ND & ND \\
\hline & - Morar em cidade grande & ND & ND & ND \\
\hline & - Escolaridade baixa & ND & ND & ND \\
\hline & - Doenças crônicas & ND & ND & ND \\
\hline & - Limitação funcional & ND & ND & ND \\
\hline & - Déficits cognitivoS & ND & ND & ND \\
\hline & - Baixo suporte social & ND & ND & ND \\
\hline & - Lócus de Controle externo ${ }^{d}$ & ND & ND & ND \\
\hline $\begin{array}{l}\text { 9. Geerlings et al, } \\
2001^{35}\end{array}$ & - Sintomas de dor & 1.57 & $1.29-1.92$ & 0.001 \\
\hline \multirow{9}{*}{$\begin{array}{l}\text { 10. Heun, Heun, } \\
2005^{36}\end{array}$} & - Idade & $\mathrm{RR}$ & IC & $\mathrm{P}$ \\
\hline & - Sexo feminino & 1.02 & $1.01-1.04$ & $<0.001$ \\
\hline & - Episódio depressivo prévio & 2.1 & $1.6-2.74$ & $<0.001$ \\
\hline & - Demência & 260 & $150-450$ & $<0.001$ \\
\hline & - Dificuldades cognitivas leves & 2.1 & $1.5-3.0$ & $<0.001$ \\
\hline & - Dificuldades cognitivas subjetivas & 1.8 & $1.4-2.5$ & $<0.001$ \\
\hline & - Ansiedade & 2.8 & 2.1-3.6 & $<0.001$ \\
\hline & - Transtor nos somatoformes & 3.6 & $2.5-5.1$ & $<0.001$ \\
\hline & & 3.0 & $1.8-4.8$ & $<0.001$ \\
\hline \multirow{17}{*}{$\begin{array}{l}\text { 11. Harris et al, } \\
2006^{37}\end{array}$} & - Idade avançada ( $\geq 85$ anos) & OR & IC & $\mathrm{P}$ \\
\hline & - Episódio depressivo prévio & 5.7 & $2.2-14.7$ & ND \\
\hline & - Ansiedade & 2.1 & $1.7-2.5$ & ND \\
\hline & - Condição de saúde (regular a ruim) & 4.4 & $1.7-11.3$ & ND \\
\hline & - Piora da condição da saúde & 9.3 & $5.3-16.4$ & ND \\
\hline & - Limitação funcional & 2.9 & $1.6-5.6$ & ND \\
\hline & - Dor & 4.7 & $1.6-14.1$ & ND \\
\hline & - Gravidade da doença & 6.0 & $3.5-10.3$ & ND \\
\hline & - Dificuldades com visão & 3.2 & $1.7-6.2$ & ND \\
\hline & - Solidão & 3.4 & $1.6-7.1$ & ND \\
\hline & - Frequentemente só & 3.6 & $2.0-6.3$ & ND \\
\hline & - Insatisfação com suporte social recebido & 2.5 & $1.5-4.1$ & ND \\
\hline & - Conflitos nos relacionamentos pessoais & 5.5 & $3.2-9.6$ & ND \\
\hline & - Problemas financeiros & 2.4 & $1.2-4.8$ & ND \\
\hline & - Eventos de vida (doença/lesão) & 3.8 & $1.7-9.0$ & ND \\
\hline & - Eventos de vida (mudança para casa de saúde) & 2.7 & $1.6-4.5$ & ND \\
\hline & & 2.6 & $1.2-5.7$ & ND \\
\hline
\end{tabular}

$\mathrm{ND}=$ dados não disponíveis; ${ }^{2}$ Deficiência (impairment): perda ou anormalidade de estrutura ou função psicológica, fisiológica ou anatômica, temporária ou permanente; ${ }^{b}$ AVD's: Atividades de vida diária; 'Desvantagem (handicap): prejuízo resultante de deficiência ou incapacidade, que limita ou impede o desempenho de papéis. ${ }^{\mathrm{d}}$ neuroticismo $=$ tendência que algumas pessoas têm para serem nervosas, ansiosas, de humor variável, susceptíveis e, muitas vezes emocionalmente instáveis. ${ }^{\mathrm{d} L o c u s}$ de controle $=$ traço de personalidade que marca vulnerabilidade para depressão e ansiedade, locus de controle externo = caracteriza aqueles indivíduos que tendem a se sentir vítimas do destino e são dependentes de intervenção da sorte ou outras pessoas ou seres mais poderosos. 
Tabela 4 - Comparação dos estudos quanto à presença dos fatores de risco associados com depressão. São Paulo, SP, 2007.

\begin{tabular}{|c|c|c|c|c|c|c|c|c|c|c|c|c|}
\hline Estudo Fator & $1^{27}$ & $2^{28}$ & $3^{29}$ & $4^{30}$ & $5^{31}$ & $6^{32}$ & $7^{33}$ & $8^{34}$ & $9^{35}$ & $10^{36}$ & $11^{37}$ & $\begin{array}{c}\text { Total } \\
\text { por } \\
\text { estudo }\end{array}$ \\
\hline Sexo Feminino & $\mathrm{X}$ & $\#$ & $\#$ & $\mathrm{X}$ & $\#$ & $\#$ & $\mathrm{X}$ & $\mathrm{X}$ & & $\mathrm{X}$ & 0 & 5 \\
\hline Idade & $\mathrm{X}$ & $\#$ & $\#$ & $\mathrm{X}$ & $\mathrm{X}$ & 0 & $\mathrm{X}$ & $\mathrm{X}$ & & $\mathrm{X}$ & $\mathrm{X}$ & 7 \\
\hline Condição marital & $\#$ & $\mathrm{X}$ & & $\mathrm{X}$ & $\#$ & & \# & $\mathrm{X}$ & & & & 3 \\
\hline Escolaridade & $\#$ & & & $\mathrm{X}$ & $\#$ & $\#$ & $\mathrm{X}$ & $\mathrm{X}$ & & $\#$ & & 3 \\
\hline $\begin{array}{l}\text { Condição sócio- } \\
\text { econômica }\end{array}$ & $\mathrm{X}$ & \# & & & $\#$ & & $\mathrm{X}$ & & & & $\mathrm{X}$ & 3 \\
\hline Condições de Moradia & $\mathrm{X}$ & & & & & & & $\mathrm{X}$ & & & & 2 \\
\hline Suporte social & $\mathrm{X}$ & $\mathrm{X}$ & & & $\#$ & $\#$ & $\mathrm{X}$ & $\mathrm{X}$ & & & $\mathrm{X}$ & 5 \\
\hline $\begin{array}{l}\text { Eventos de vida } \\
\text { estressores }\end{array}$ & $\mathrm{X}$ & \# & $\mathrm{X}$ & & $\mathrm{X}$ & $\mathrm{X}$ & & & & & $\mathrm{X}$ & 5 \\
\hline $\begin{array}{l}\text { Histórico psiquiátrico } \\
\text { prévio }\end{array}$ & $\mathrm{X}$ & & $\#$ & & $\mathrm{X}$ & & & & & $\mathrm{X}$ & $\mathrm{X}$ & 4 \\
\hline $\begin{array}{l}\text { Histórico psiquiátrico } \\
\text { familiar }\end{array}$ & & & & & $\#$ & & & & & & & 0 \\
\hline Comorbidades psiquiátricas & & & & & $\mathrm{X}$ & & & & & $\mathrm{X}$ & $\mathrm{X}$ & 3 \\
\hline Personalidade & & & & & & $\mathrm{X}$ & & $\mathrm{X}$ & & & \# & 2 \\
\hline Uso/abuso de álcool & & & & & & $\#$ & $\#$ & & & $\#$ & & 0 \\
\hline Distúrbios do sono & & $\mathrm{X}$ & & & & $\#$ & $\mathrm{X}$ & & & & & 2 \\
\hline Déficits cognitivos & $\mathrm{X}$ & $\#$ & & & $\#$ & & & $\mathrm{X}$ & & $\mathrm{X}$ & & 3 \\
\hline Doenças crônicas & $\mathrm{X}$ & $\mathrm{X}$ & & $\mathrm{X}$ & $\mathrm{X}$ & $\#$ & & $\mathrm{X}$ & & & & 5 \\
\hline Doenças agudas & & & & & & $\mathrm{X}$ & & & & & & 1 \\
\hline Comorbidades orgânicas & $\mathrm{X}$ & $\mathrm{X}$ & & $\mathrm{X}$ & $\mathrm{X}$ & & & & & & $\mathrm{X}$ & 5 \\
\hline Gravidade da doença & & $\mathrm{X}$ & & & & & & & & & $\mathrm{X}$ & 2 \\
\hline Limitação funcional & $\mathrm{X}$ & $\mathrm{X}$ & & $\mathrm{X}$ & $\mathrm{X}$ & $\#$ & $\mathrm{X}$ & $\mathrm{X}$ & & & $\mathrm{X}$ & 7 \\
\hline Dor & & $\mathrm{X}$ & & & & $\mathrm{X}$ & & & $\mathrm{X}$ & & $\mathrm{X}$ & 4 \\
\hline
\end{tabular}

$\mathrm{X}=$ indica fator de risco; 0 = indica fator protetor; $\#=$ indica que o fator não apresentou nenhum efeito; espaço em branco indica que o fator não foi avaliado.

\section{DISCUSSÃO}

A incidência de casos de depressão e sintomas depressivos apresentou uma variação ampla entre os estudos realizados, de $5,4 \%$ a $24,15 \%$ (tabela 2).

Houve discrepância de resultados, mesmo naqueles estudos que usaram critérios padronizados e amplamente reconhecidos para depressão maior, como foi observado nos estudos de Roberts et al. ${ }^{27}$ e Heun. ${ }^{36}$

A variação entre as taxas pode ser atribuída à utilização de diferentes escalas de medida, definições de depressão, métodos de aplicação do instrumento, populações de interesse, tempos de seguimento e contextos culturais. 
Em alguns estudos, a perda de seguimento de pessoas esteve associada com quadros depressivos no início do estudo, o que pode indicar uma subestimação da incidência de depressão. ${ }^{28,30,32,37}$

Em relação aos fatores de risco, foram identificados 19 fatores preditores de quadros depressivos em idosos residentes na comunidade: sexo feminino, idade avançada, situação conjugal (ser solteiro, separado/divorciado), baixa escolaridade, condição socioeconômica desfavorável, condições de moradia inadequadas, baixo suporte social, presença de eventos de vida estressores, histórico psiquiátrico prévio, presença de comorbidades psiquiátricas, características de personalidade, distúrbios do sono, déficits cognitivos, doenças crônicas e agudas, co-morbidades orgânicas, gravidade da doença, limitação funcional e dor.

Apesar de a associação entre depressão e sexo feminino ter aparecido em alguns estudos, ${ }^{27,30,33,34,36}$ outros não foram capazes de demonstrar que as mulheres idosas estavam sob maior risco. ${ }^{28,29,31,32,37}$

A típica "depressão feminina” parece ser decorrente mais da exposição frequente da mulher idosa a alguns fatores de risco (condição marital, baixa renda e escolaridade, condição de saúde, limitação funcional, baixo suporte social) do que uma questão meramente biológica. ${ }^{38}$

Sete estudos evidenciaram a associação entre a idade avançada e a incidência aumentada de depressão, ${ }^{27,30,31,33,34,36,37}$ mas três es- tudos ${ }^{27,28,31}$ constataram que os idosos que tinham idade mais avançada e eram saudáveis, livres de incapacidades e de limitações não diferiam em relação à depressão dos idosos mais jovens. ${ }^{27,28}$

As últimas evidências sugerem que a incidência de depressão pode estar provavelmente associada à vulnerabilidade dos idosos para problemas de saúde e suas limitações do que à idade propriamente dita. ${ }^{28}$ Estas considerações podem ser corroboradas, ainda, pela associação verificada entre incidência aumentada de depressão com presença de doenças crônicas, ${ }^{27,28,30-35,37}$ limitação funcional decorrente destas doenças ${ }^{27,28,30,31,33,34,35,37}$ e existência de déficits $\operatorname{cog}$ nitivos..$^{27,34,36} \mathrm{De}$ acordo com os achados de Prince et al. ${ }^{28}$ incapacidade, principalmente prejuízos no desempenho dos papéis que o indivíduo exerce em seu meio, foi o principal preditor de depressão geriátrica.

Os idosos deprimidos avaliados em três estudos apresentavam condições crônicas de saúde e incapacidades que poderiam estar contribuindo para a ocorrência de recaídas ou, mesmo, tornar crônicos os quadros depressivos. . $^{27,31,37}$

Suporte social e as características de personalidade exercem papel importante na forma com que o indivíduo lida com os estressores psicossociais. ${ }^{27,28,29,31,34,37}$ Schoevers et al. ${ }^{31}$ observaram que a dependência de outras pessoas resultante de eventos de vida negativos (por exemplo, doenças, limitação funcional e perda do cônjuge) pode ser reduzida pela situação conjugal ou suporte 
social adequado. De outra forma, em idosos, o impacto dos eventos estressores na incidência de depressão pode ser modificado em função da vulnerabilidade ambiental e individual. . $^{31,32,34}$

Turvey et al..$^{29}$ observaram que, após dois anos da morte do parceiro, cerca de $12 \%$ dos idosos continuavam com escores altos de sintomas depressivos, independente de sexo e idade. A dificuldade de adaptação destes indivíduos à perda pode estar relacionada tanto a adversidades ambientais ${ }^{31}$ quanto à vulnerabilidade individual..$^{31,32,34}$

Outro importante preditor de depressão foi a ocorrência de episódios depressivos prévios na vida dos indivíduos deprimidos, ${ }^{27,31,36,37}$ o que suporta a hipótese de que depressão pode ser uma condição crônica ou recorrente..$^{27,37}$

O reconhecimento e tratamento de transtornos depressivos na população idosa permanecem um desafio. Os esforços no sentido de identificar os fatores de risco associados com o surgimento de depressão em idosos vivendo na comunidade podem colaborar para a construção de estratégias amplas de intervenção por equipes multiprofissionais.

Estratégias de intervenção mais precoces e adequadas só podem acontecer se os profissionais que atendem idosos estiverem capacitados para identificar e tratar ou pelo menos encaminhar os casos que demandem tratamentos especializados.

Lamentavelmente, todos os artigos de coorte longitudinal localizados, para fins deste trabalho, foram realizados em países de Primeiro Mundo. Nenhum foi realizado nos países de origem hispânica e nem no Brasil. Portanto, os dados aqui descritos não podem ser tomados como referências para nossa população idosa, uma vez que há diferenças socioeconômicas e culturais importantes a serem consideradas.

\section{CONSIDERAÇÕES FINAIS}

A incidência de depressão na população idosa residente na comunidade é $12 \%$. Os preditores de depressão identificados em idosos vivendo na comunidade foram: sexo feminino, idade avançada, condição marital, baixa escolaridade, condição socioeconômica desfavorável, condições de moradia, baixo suporte social, presença de eventos de vida estressores, histórico psiquiátrico prévio, presença de comorbidades psiquiátricas, características de personalidade, distúrbios do sono, déficits cognitivos, doenças crônicas e agudas, co-morbidades orgânicas, gravidade da doença, limitação funcional e dor.

Conclui-se pela necessidade de realizar, em nosso meio, estudos com desenhos adequados, como o coorte longitudinal, que possibilitem identificar a incidência de depressão e os fatores de risco associados na população idosa brasileira.

A identificação pode favorecer a geração de políticas públicas que propiciem intervenções mais precoces e adequadas pelos profissionais envolvidos na prestação de serviços para essa população. 


\section{REFERÊNCIAS}

1. IBGE. Censo Demográfico 2000. [acesso em: 20 ago. 2007].Disponível em: URL: http://www.ibge.gov.br/censo.

2. IBGE. Síntese de indicadores sociais 2005 . [acesso em: 20 ago. 2007].Disponível em: http://www.ibge.gov.br/ sinteseindicsociais2005.

3. Camarano AA, Kanso S, Pasinato MT, Mello JL. Idosos brasileiros: indicadores de condições de vida e de acompanhamento de políticas. Brasília: Subsecretaria de Direitos Humanos; 2005.

4. Papaléo Netto M. O estudo da velhice no século XX: histórico, definição do campo e termos básicos. In: Freitas EV, et al. Tratado de Geriatria e Gerontologia. Rio de Janeiro: Guanabara Koogan; 2006. p. 2-12.

5. Garcez-Leme LE, Leme MD, Espino D. Geriatrics in Brazil: a big country with big opportunities. JAGS 2005; 53 (11):2018-22.

6. Nunes, A. Aspectos sobre a morbidade dos idosos no Brasil. Como vai? População brasileira 1999; 4(2): 20-32.

7. Stoppe Jr A, Louzã Neto MR. Depressão na terceira idade. São Paulo: Lemos Editorial; 1999.

8. Garrido R, Menezes PR. O Brasil está envelhecendo: boas e más notícias por uma perspectiva epidemiológica. Rev Bras Psiquiatr 2002; 24 (supl 1): 3-6.

9. Murray CJ, Lopez AD. The global burden of disease. Geneva: World Health Organization; 1996.

10. Irwin $\mathrm{M}$, Artin $\mathrm{KH}, \mathrm{Oxman} \mathrm{MN}$. Screening for depression in the older adult. Arch Intern Med 1999;159:1701-4.

11. Blay SL. Características clínicas, fatores de risco e cursos da depressão em idosos. In: Lafer B, Almeida OP, Fráguas Jr R,
Miguel EC. Depressão no ciclo da vida. São Paulo: Artes Médicas; 2000.p. 45-53.

12. Del Porto JA. Conceito de depressão e seus limites. . In: Lafer B, Almeida OP, Fráguas Jr R, Miguel EC. Depressão no ciclo da vida. São Paulo: Artes Médicas; 2000. p. 20-8.

13. DSM-IV-TR. Manual Diagnóstico e Estatístico de Tratamento de Transtornos Mentais (2002). Porto Alegre: Artes Médicas; 2002.

14. Classificação de Transtornos Mentais e de Comportamento da CID-10 (1993). Porto Alegre: Artes Médicas; 1993.

15. DSM-IV. Manual Diagnóstico e Estatístico de Tratamento de Transtornos Mentais (1994). 4 ed. Porto Alegre: Artes Médicas; 2000.

16. Reynolds CF, Kupfer DJ. Depression and aging: a look to the future. Psychiatr Serv 1999; 50:1167-72.

17. Koenig HG, Meador KG, Shelp F, Goli V, Cohen HJ, Blazer DG. Major depressive disorder in hospitalized medical ill patients: an examination of young and elderly male veterans. J Am Geriatr Soc 1991; 39:881-90.

18. NIH Consensus Conference. Diagnosis and treatment of depression in late life. JAMA. 1992; 268 (8):1018-24.

19. Hay DP, Franson KL, Hay L, Grossberg GT. Depression. In: Duthie EH, Katz PR. Practice of Geriatrics. 3rd. ed. Philadelphia: W.B. Saunders Company; 1998. p. 286-94.

20. Lebowitz BD, Pearson JL, Schneider LS, Reynolds CF 3d, Alexopoulos GS, Bruce $\mathrm{ML}$, et al. Diagnosis and Treatment of depression in late life: Consensus 
Statement Update. JAMA 1997; 278(14):1186-90.

21. Silverstone PH. Depression increases mortality and morbidity in acute lifethreatening medical illness. J Psychosom Res 1990; 34:651-7.

22. Katona C; Livingston G. Comorbid depression in older people. London: Martin Dunitz; 1997.

23. Cole GC, Dendukuri N. Risk factors for depression among elderly community subjects: a systematic review and metaanalysis. Am J Psychiatry 2003;160: 1147-56.

24. Cankurtaran M, Halil M, Yavuz B, Dagli $\mathrm{N}$, Cankurtaran E, Ariogul S. Depression and concomitant diseases in a Turkish geriatric outpatient setting. Arch Gerontol Geriatr 2005; 40 (3): 307-15.

25. Djernes JK. Prevalence and predictors of depression in populations of elderly: a review. Acta Psychiatr Scand 2006;113: 372-87.

26. Jonge P, Kempen G, Sanderman R, Ranchor A, van Jaarsveld C, van Sonderen E et al. Depressive symptoms in elderly patients after a somatic illness event: prevalence, persistence and risk factors. Psychosomatics 2006;47: 33-42.

27. Roberts RE, Kaplan GA, Shema SJ, Strawbridge WJ. Does growing old increase the risk for depression? Am J Psychiatry 1997;154:1384-90.

28. Prince MJ, Harwood RH, Thomas A, Mann AH. A prospective populationbased cohort study of the effects of disablement and social milien on the onset and maintenance of late-life depression. The Gospel Oak Project VII. Psychol Med 1998; 28: 337-50.

29. Turvey CL, Carney C, Arndt S, Wallace RB, Herzog R. Conjugal loss and syndromal depression in a sample of elders aged 70 years or older. Am J Psychiatry 1999;156:1596-601.

30. Geerlings SW, Beekman AT, Deeg DJ, van Tilburg W. Physical health and the onset and persistence of depression in older adults: an eight-wave prospective community-based study. Psychol Med 2000; 30(2): 369-80.

31. Schoevers RA, Beekman AT, Deeg DJ, Geerlings MI, Jonker C, van Tilburg W. Risk factors for depression in later life: results of a prospective community based study (AMSTEL). J Affect Disord 2000; 59(2):127-37.

32. Livingston G, Watkin V, Milne B, Manela MV, Katona C. Who becomes depressed? The Islington community study of older people1. J Affect Disord 2000; 58 (2):125-33.

33. Roberts RE, Shema SJ, Kaplan GA, Strawbridge WJ. Sleep complaints and depression in an aging cohort: a prospective perspective. Am J Psychiatry 2000;157(1): 81-8

34. Beekman AT, Deeg DJ, Geerlings SW, Schoevers RA, Smit JH, van Tilburg W. Emergence and persistence of late life depression: a 3-year follow-up of the Longitudinal Aging Study Amsterdam. J Affect Disord 2001; 65 (2):131-8.

35. Geerlings SW, Twisk JW, Beekman AT, Deeg DJ, van Tilburg W. Longitudinal relationship between pain and depression in older adults: sex, age and physical disability. Soc Psychiatry Psychiatr Epidemiol 2002; 37: 23-30.

36. Heun R, Hein S. Risk factors of major depression in the elderly. Eur Psychiatry 2005; 20 (3):199-204.

37. Harris T, Cook DG, Victor C, DeWilde S, Beighton C. Onset and persistence of depression in older people-results from a 2-year community follow-up study. Age Ageing. 2006; 35(1): 25-32. 
38. Sonnenberg CM, Beekman ATF, Deeg DJ, van Tilburg W. Sex differences in late-life depression. Acta Psychiatr Scand 2000;101:286-92.

Recebido: 14/7/2008

Reapresentado: 10/10/2008

Aprovado: 21/10/2008 